\title{
Tobacco use and betel quid chewing among adults in Myanmar- estimates and social determinants from demographic and health survey, 2015-16
}

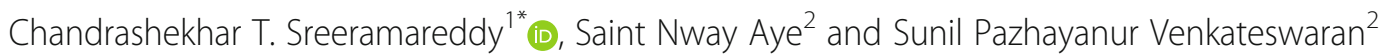

\begin{abstract}
Background: National-level prevalence of tobacco use and betel quid chewing, and associated socio-demographic factors were estimated using first-ever, Myanmar Demographic Health Survey, 2015-16.

Methods: Questions about tobacco smoking, smokeless tobacco use, and betel quid chewing were used to create outcome variables such as tobacco smoking, smokeless tobacco use, and 'dual use' (tobacco use and betel quid chewing). Sex-stratified weighted prevalence rates, distribution by socio-demographic factors were presented. Association of demographic factors with tobacco and/or betel quid chewing was assessed by multinomial logistic regression.

Results: Among men, prevalence (\%) of tobacco use and betel quid chewing was 40.9 ( $95 \% \mathrm{Cl} 38.1,42.1)$ and 58.9 $(95 \% \mathrm{Cl} 56.3,61.6)$ respectively. Among women tobacco use was $3.7(95 \% \mathrm{Cl} 2.0,4.3)$ and betel quid chewing 18.2 $(95 \% \mathrm{Cl} 16.4,20.0)$. Among men prevalence of either tobacco or betel quid and 'dual use' was 50.4 (95\% Cl 48.5, $52.3)$ and $25.0(95 \% \mathrm{Cl} 23.1,26.8)$ respectively, whereas among women the corresponding rates were $17.9(95 \% \mathrm{Cl}$ $16.2,19.6)$ and $2.0(95 \% \mathrm{Cl} 1.6,2.9)$. Smokeless tobacco use was low $(<5 \%)$ in both sexes. Tobacco use and/or betel quid chewing was associated with age, wealth, marital status, and occupation in both sexes. However, the effect sizes were much larger among women for wealth groups. People of older age and lower wealth had a higher odds of being a tobacco user and/or betel quid chewer.

Conclusions: In Myanmar, prevalence of both tobacco use and betel quid chewing was high particularly among men. Tobacco control interventions should be strictly implemented and 'dual use' of both tobacco and betel quid should also be urgently addressed.
\end{abstract}

Keywords: Tobacco use, Betel quid chewing, Prevalence, Myanmar

* Correspondence: chandrashekharats@yahoo.com

'Department of Community Medicine, International Medical University, Bukit Jalil, 57000 Kuala Lumpur, Malaysia

Full list of author information is available at the end of the article

C C The Author(s). 2021 Open Access This article is licensed under a Creative Commons Attribution 4.0 International License, which permits use, sharing, adaptation, distribution and reproduction in any medium or format, as long as you give appropriate credit to the original author(s) and the source, provide a link to the Creative Commons licence, and indicate if changes were made. The images or other third party material in this article are included in the article's Creative Commons licence, unless indicated otherwise in a credit line to the material. If material is not included in the article's Creative Commons licence and your intended use is not permitted by statutory regulation or exceeds the permitted use, you will need to obtain permission directly from the copyright holder. To view a copy of this licence, visit http://creativecommons.org/licenses/by/4.0/. The Creative Commons Public Domain Dedication waiver (http://creativecommons.org/publicdomain/zero/1.0/) applies to the data made available in this article, unless otherwise stated in a credit line to the data. 


\section{Background}

Tobacco smoking still stands as the second leading risk factor for disability and death worldwide, despite several evidence-based, anti-smoking interventions [1]. Global prevalence of smoking was estimated at 25\% in 2015 with very little decline since 1990 [2, 3]. Smoking prevalence has shown wide sex-wise and geographic differentials and disease burden attributable to smoking is growing in low-income countries [4]. Southeast Asia (SEA) region is home to an estimated 400 million tobacco users causing an estimated 1.2 million smoking attributable deaths annually [4]. Tobacco is also consumed in diverse smokeless forms in South Asia (SA) and SEA [4-6]. Smokeless tobacco (SLT) use is a well-established risk factor for oropharyngeal cancers [7]. In addition, betel quid chewing with or without tobacco leaves along with other varied ingredients is a widely prevalent practice in many parts of Asia including SEA [8]. Betel quid chewing mixed with tobacco greatly increases the risk for bleeding gums, periodontal diseases, oral lesions and oral cancer [8-12].

Tobacco use is common is Myanmar where 'cheroots' are also smoked in addition to manufactured cigarettes. Cheroot is a filterless indigenous cigar of Myanmar which has both its ends truncated. Cheroots are hand rolled using bark, stems, roots and sundry leaves. They are filled with a choice blend of tobacco and tied with a red silk thread. Smokeless tobacco use is also very common in Myanmar where eating raw and cured tobacco leaves is most common but tobacco leaves are also mixed with substances such as alcohol, honey, lime etc. $[13,14]$. Betel quid chewing is very common in Myanmar as in other SA countries. Kun-ywet is the Myanmar term for betel leaf, areca nut is called Kunthee, and the preparation is called Kun-yar. Offering and chewing Kun-yar or betel quids is ceremonial and deeply rooted in the traditional culture of Myanmar, like other countries in SA. Betel quid chewers usually do not add tobacco into the betel quid during the early days. However, at later stages some betel quid chewers mix tobacco leaves into the quid and gradually develop nicotine dependence. In Myanmar, different forms of tobacco flavours, and contents in the betel quid is practiced widely and is believed to be breath-freshening and a mouth-cleansing agent $[13,14]$. Research has shown that about $80 \%$ of betel quid chewers add tobacco leaves and $9.6 \%$ of them had premalignant oral disorders [12].

The Framework Convention on Tobacco Control (FCTC) adopted in 2003 has been ratified by more than 180 countries worldwide [15]. Under the FCTC, monitoring of worldwide use of tobacco by population-based surveys has been prioritized to understand the patterns of tobacco use behaviors, assess the impact of tobacco control measures, and to assist policy changes in tobacco control. Consumption of both smoking and smokeless tobacco products is being recognised as a distinct tobacco use behaviour to combat the tobacco epidemic since it increases the risk of tobacco-induced diseases [16]. Smoking, SLT and betel quid chewing all are known risk factors for oropharyngeal cancers, which may act additively or synergistically [7-10, 17]. In SEA, SLT use is increasing $[5,18]$ and SLT has been used as a replacement to smoking and tobacco users are also known to switch between these products in an attempt to quit $[19,20]$. Studying socio-economic determinants of tobacco use and betel quid chewing also helps to develop targeted interventions towards vulnerable and disadvantaged populations [6]. National-level prevalence of smoking and SLT in SEA are well reported $[5,6]$ but very few reports are available on betel quid chewing in Myanmar [21, 22]. However, there is a lack of nationally representative surveys for Myanmar reporting on both tobacco use and betel quid chewing. Myanmar participated in its first Demographic and Health Survey (DHS) in 2015-16. We aimed to provide national level estimates for prevalence of tobacco use (smoking and SLT), betel quid chewing and 'dual use'. We also aimed to assess the socio-demographic determinants of 'dual use' (both tobacco use and betel quid chewing).

\section{Methods}

\section{Data source}

The 2015-16 Myanmar Demographic and Health Survey (2015-16 MDHS) was implemented by the Ministry of Health and Sports (MoHS) between December 7, 2015, and July 7, 2016. MDHS was funded by the United States Agency for International Development (USAID) and the Three Millennium Development Goal Fund (3MDG) with technical support from Inner City Fund (ICF International, Inc.) via the DHS Program. The primary objective of the 2015-16 MDHS was to provide up-to-date estimates of basic demographic and health indicators such as fertility, family planning, health, nutrition and so on [23].

\section{Sampling and sample size}

The sampling frame for MDHS consisted of 76,990 primary sampling units (PSUs) spread across the country. A PSU was either a census enumeration area (EA) or a ward or village tract. Geographic locations where internal conflict was ongoing were considered as sensitive areas and were not enumerated during the census. Each PSU had cartographic materials that delineated its geographic location, boundaries, main access points, and landmarks. The sampling frame provided information about administrative subordination by state, region or district, urban or rural, and the estimated number of residential households in each PSU. Institutional 
populations, such as hotels, barracks, and prisons were not sampled. However, internally-displaced population camps were included [23]. All men and women aged 15-49 years who were permanent residents of the selected households or visitors who stayed in the households the night before the survey date were interviewed. Men were interviewed in only half of the selected households [23].

The final samples of households in MDHS were selected by stratified two-stage random sampling method. Stratification by urban-rural was done and clusters were selected by probability proportionate to size technique. Sampling weights were calculated based on sampling probabilities calculated separately for each sampling stage and for each cluster. The household head provided responses to the general questions about the household and listed all the members of the household. Men and women enlisted were eligible for the interview, if they were either permanent residents or visitors who stayed in that house the night before. Trained interviewers collected information about demographic and socioeconomic factors and health status. Questions about tobacco use and betel quid chewing were asked to all eligible men and women $[24,25]$.

\section{Outcome variables}

The following six questions were asked to gather information about tobacco use and betel quid chewing in Myanmar:

1) Do you currently smoke cigarettes? (response as 'yes' or 'no')

2) In the last $24 \mathrm{~h}$, how many cigarettes did you smoke? (response as numerical)

3) Do you currently smoke or use any other type of tobacco? (response as 'yes' or 'no')

4) What (other) type of tobacco do you currently smoke or use? (options provided were pipe, cigar, cheroot, chewing tobacco, snuff, others)

5) Do you currently chew betel nuts? (response as 'yes' or 'no')

6) In the last $24 \mathrm{~h}$, how many pieces did you chew? (response as numerical)

Each respondent was classified as 'tobacco smoker', if the response was 'yes' to the first and third questions and they responded as 'pipe,' 'cigar', or 'cheroot' to the fourth question. The respondents were classified as 'SLT user' if the response to the fourth question was any form of SLT, including 'chewing tobacco, 'and 'snuff'. If they responded as 'yes' to fifth question, classified as 'betel quid chewer'. To assess the socio-demographic determinants, we created a nominal outcome variable as 'nonuser', either 'tobacco user' (any type of smoking or smokeless tobacco) or 'betel quid chewer' and 'dual users' (both tobacco use and betel quid chewing).

\section{Explanatory variables}

For multinomial logistic regression analyses, we used age in completed years, marital status, education, occupation, wealth index, and questions on weekly frequency of exposure to mass media (1) reading newspaper or magazine, 2) listening to radio and 3) watching television). Exposure to these media was measured as 'at least once a week', 'less than once a week' and 'not at all' and we scored these responses as 2,1 and 0 respectively. We generated a score by summing up the responses to the three questions to quantify the frequency of exposure to mass media. The possible scores ranged from 0 to 6 . Marital status was classified as 'never married', 'currently married' and 'single'. 'Single' constituted being separated, divorced, and widowed. Educational level was classified as 'no education', 'primary', 'secondary,' or 'higher.' Household wealth index, considered as a reliable proxy for household economic status [26] was calculated based on a standard set of household assets, dwelling characteristics, and ownership of consumer items as observed by the interviewer. Participants were ranked on the basis of their household wealth score by dividing them into quintiles where the first quintile was the poorest $20 \%$ of the households and the fifth quintile was the wealthiest $20 \%$ [27]. Spatial variables such as urban-rural, and state or region or district were not used in the multinomial logistic regression analyses since the sample selection was stratified by urban-rural and the sample distribution by administrative units (states/regions) was similar.

\section{Ethics statement}

The institutional review boards of ORC Macro International Inc. and participating institutions in Myanmar provided ethical clearance for MDHS. In each survey, participants were informed about voluntary participation and confidentiality of information and they could refrain from responding to any of the questions. Before each interview, details of the survey were explained, and informed consent was obtained. Written consent was not obtained since no intervention was applied to the participants.

\section{Data analysis}

All analyses were stratified by sex. Unweighted and weighted proportions and weighted prevalence rates for types of tobacco smoking, SLT use and betel quid chewing were estimated. Sample weights were applied during estimation to account for the complex sampling design used in MDHS. Weighted prevalence estimates of tobacco smoking, SLT use and betel quid chewing were calculated by the age groups, marital status, occupation, 
education, and wealth quintiles. Multinomial logistic regression analyses were done to assess socio-demographic factors associated with 'dual use', tobacco use, betel quid chewing as compared to non-user (reference category). Using 'svy' command on STATA/IC version 11.2 the multinomial logistic regression model we included for age, wealth, marital status, occupation and score for exposure to mass media in the models. However, education was not included in the full model since it was correlated with wealth and occupation. Adjusted odds ratios their 95\% confidence intervals, and $p$-values were calculated.

\section{Results}

Sociodemographic characteristics of survey population Sociodemographic characteristics presented as both weighted and unweighted numbers and proportions are shown in Table 1. A total of 17,622 subjects were surveyed of whom $27 \%$ were men and $73 \%$ were women and their mean age was 31.3 years and 31.6 years respectively. More than half of respondents were aged between 20 and 40 years. More than two-thirds of respondents were rural residents. About $80 \%$ of men and women were educated up to primary and secondary level. About a third were never married and $61 \%$ were currently married. Men and women were nearly equally distributed by household wealth quintiles. Men were mostly working in agricultural and manual work (77.0\%) and women were unemployed/domestic workers (28.8\%) and manual workers (29.8\%).

\section{Sex-wise distribution of types of tobacco products and betel quid chewing}

Unweighted and weighted numbers and their proportions of types of tobacco products consumed for multiple

Table 1 Sociodemographic characteristics of survey population

\begin{tabular}{|c|c|c|c|c|}
\hline & Men $(N=4737)$ & & Women $(N=12,885)$ & \\
\hline & Unweighted Number (\%) & Weighted Number (\%) & Unweighted Number (\%) & Weighted Number (\%) \\
\hline Age & $\begin{array}{l}\text { Mean }=31.27(10.1) \\
\text { Median }=31(22,40)\end{array}$ & & $\begin{array}{l}\text { Mean }=31.6(9.88) \\
\text { Median }=32(23,40)\end{array}$ & \\
\hline Age groups & & & & \\
\hline $15-20$ & $926(19.5)$ & $891(18.8)$ & $2220(17.3)$ & $2205(17.1)$ \\
\hline $21-30$ & $1380(29.1)$ & $1373(29.0)$ & $3815(29.6)$ & $3771(29.3)$ \\
\hline $31-40$ & $1315(27.8)$ & $1351(28.5)$ & $3843(29.8)$ & $3929(30.5)$ \\
\hline $41-49$ & $1116(23.6)$ & $1122(23.7)$ & $3007(23.3)$ & $2980(23.1)$ \\
\hline Education & & & & \\
\hline No education & $559(11.8)$ & $576(12.2)$ & $1592(12.0)$ & $1606(12.5)$ \\
\hline Primary & $1630(34.4)$ & $1684(35.5)$ & $5129(40.0)$ & $5305(41.2)$ \\
\hline Secondary & $2224(47.0)$ & $2139(45.1)$ & $4838(37.7)$ & $4647(36.0)$ \\
\hline Higher & $324(6.8)$ & $340(7.2)$ & $1324(10.3)$ & $1325(10.3)$ \\
\hline Marital status & & & & \\
\hline Never married & 1695 (35.9) & $1645(34.8)$ & $4146(32.3)$ & $4273(33.3)$ \\
\hline Currently married & $2916(61.7)$ & $2955(62.6)$ & $7870(61.4)$ & $7751(60.4)$ \\
\hline Divorced/Separated/Widowed & $114(2.4)$ & $125(2.6)$ & $811(6.3)$ & $803(6.3)$ \\
\hline Wealth Index & & & & \\
\hline Poorest & $904(19.1)$ & $890(18.8)$ & $2364(18.3)$ & $2274(17.6)$ \\
\hline Poorer & $933(19.7)$ & $916(19.3)$ & $2451(19.0)$ & $2408(18.7)$ \\
\hline Middle & $1016(21.4)$ & $980(20.7)$ & $2633(20.4)$ & $2633(20.4)$ \\
\hline Richer & $995(21.0)$ & $986(20.8)$ & $2739(21.3)$ & $2702(21.0)$ \\
\hline Richest & $889(18.8)$ & $965(20.4)$ & $2698(21.0)$ & $2868(22.3)$ \\
\hline Type of residence & & & & \\
\hline Rural & $3416(72.1)$ & $3387(71.5)$ & $9100(70.6)$ & $9117(70.8)$ \\
\hline Urban & $1321(27.9)$ & $1351(28.5)$ & $3785(29.4)$ & $3768(29.2)$ \\
\hline Occupation & & & & \\
\hline Unemployed/domestic work & $291(6.2)$ & $295(6.2)$ & $3711(28.8)$ & $3550(27.6)$ \\
\hline Professional/service/sales & $787(16.6)$ & $820(17.3)$ & $3429(26.6)$ & $3317(25.8)$ \\
\hline Agricultural work & $1256(26.6)$ & $1274(27.0)$ & $1880(14.6)$ & $1847(14.4)$ \\
\hline Manual worker & $2394(50.6)$ & $2340(49.5)$ & $3835(29.8)$ & $4141(32.2)$ \\
\hline
\end{tabular}


tobacco products are shown in Table 2. More than half of the men $(59 \%)$ chewed betel quid, about a third (31.7\%) of the men smoked cigarettes and $14.4 \%$ of men smoked pipe/cigar/cheroot, whereas users of other types of tobacco products was less than $10 \%$. Overall, $40 \%$ of men were smoking tobacco while only $2 \%$ used smokeless tobacco. Nearly $50 \%$ the men either chewed betel quid or consumed tobacco products, while $25 \%$ of them consumed both. Only $24.6 \%$ of the men were non-users of either tobacco or betel quid. On the other hand, the tobacco use among women was very low (3.7\%) whereas betel quid chewing was $18.2 \%$. Compared to men, the proportion of women who smoked was low for cigarettes (1.7\%) and cheroots $(2.1 \%)$ as well as betel quid chewing (18.2\%). Overall, $3.7 \%$ of women smoked tobacco and only $0.2 \%$ used smokeless tobacco. A majority $(80.1 \%)$ of the women were non-users, about $17.9 \%$ either used tobacco or chewed betel quid and only $2.0 \%$ were 'dual users.'

\section{Distribution of weighted prevalence rates of tobacco smoking, SLT use, 'dual user' by socio-economic and demographic factors}

Distribution of weighted prevalence rates (\%) of tobacco use and betel quid chewing by socio-demographic factors are shown in Table 3. Weighted prevalence rates of tobacco use, and betel quid chewing varied by sociodemographic factors among both men and women. There was a clear gradient across education and wealth subgroups. Prevalence of tobacco use was highest among the respondents without education compared to those with higher education (50.8 vs. 30.7); from poorest households compared to the richest (45.7 vs. 36.9). Differentials by wealth for betel quid chewing and 'dual use' were similar among both men and women. However, the differentials were much wider among women. Betel quid chewing among men did not show any differentials by educational attainment. Both tobacco use and betel quid chewing rates were higher among divorced, widowed and separated men and women than never married and currently married. An exception was the highest rate of betel quid chewing rates among currently married men. Urban-rural differentials showed an inconsistent pattern among men; however, rural women had a higher prevalence of both tobacco use and betel quid chewing than their urban counterparts. Agricultural and manual workers had a higher prevalence rates of tobacco use as well as betel quid chewing in both sexes.

\section{Association of tobacco use and betel quid with socio- demographic factors}

Association of being both a tobacco user as well as a betel quid chewer ('dual use') and use of one product only as compared to non-use with socio-demographic factors was tested using multinomial logistic regression analyses and the results are shown in Table 4. In both sexes, older age was associated with being 'dual users' as well use one product only. The odds being tobacco user and/or betel quid chewer was higher in older age (adj. OR 1.0 to 1.1, $p<0.001$ ). However, frequency of exposure to media was not associated with either 'dual users' or use of one product only (except for tobacco use among men).

Among men being a tobacco user and/or betel quid chewer was associated with wealth index, marital status, and occupation. Men who were currently married had a lower odds of being 'dual users' (adj. OR 0.3 (95\% CI 0.2, $0.7)$ as compared to divorced/separated/widowed. Men who belonged to the occupational group professional/ service/sales had a lower odds of being 'dual users' (adj. OR $0.395 \%$ CI 0.2, 0.6) as compared to manual workers.

Table 2 Weighted and unweighted frequencies and proportions of various behaviors related to tobacco products and betel quid use among men and women in Myanmar, 2015-16

\begin{tabular}{|c|c|c|c|c|c|c|c|c|}
\hline & \multicolumn{4}{|c|}{ Men $=4737$} & \multicolumn{4}{|c|}{ Women = 12,598 } \\
\hline & \multicolumn{2}{|c|}{ Unweighted } & \multicolumn{2}{|c|}{ Weighted } & \multicolumn{2}{|c|}{ Unweighted } & \multicolumn{2}{|l|}{ Weighted } \\
\hline & Number & $\%$ & number & $\%$ & Number & $\%$ & Number & $\%$ \\
\hline Cigarette smoking & 1594 & 33.7 & 1504 & 31.7 & 277 & 2.2 & 214 & 1.7 \\
\hline Smoking pipe/cigar/cheroot/others & 714 & 15.0 & 683 & 14.4 & 399 & 3.1 & 266 & 2.1 \\
\hline Chewing Tobacco & 63 & 1.3 & 57 & 1.2 & 22 & 0.2 & 22 & 0.2 \\
\hline Snuff & 33 & 0.7 & 44 & 0.94 & 2 & 0.02 & 3 & 0.02 \\
\hline Betel quid chewing & 2779 & 58.7 & 2792 & 59.0 & 2661 & 20.7 & 2343 & 18.2 \\
\hline Tobacco smoking $(1+2)$ & 1976 & 41.7 & 1899 & 40.1 & 653 & 5.1 & 467 & 3.7 \\
\hline Smokeless tobacco use $(3+4)$ & 91 & 1.9 & 95 & 2.0 & 24 & 0.2 & 25 & 0.2 \\
\hline Any type of tobacco use $(1+2+3+4)$ & 2019 & 42.6 & 1940 & 40.9 & 674 & 5.2 & 491 & 3.8 \\
\hline Non-use of either tobacco or chew betel quid & 1179 & 24.9 & 1166 & 24.6 & 9887 & 76.7 & 10,316 & 80.1 \\
\hline Use of either tobacco or betel quid & 2318 & 48.9 & 2410 & 50.4 & 2660 & 20.7 & 2304 & 17.9 \\
\hline Use of both tobacco and betel quid & 1240 & 26.2 & 1161 & 25.0 & 336 & 2.6 & 263 & 2.0 \\
\hline
\end{tabular}


Table 3 Weighted prevalence rates (\%) of tobacco use, betel quid chewing and dual use by socio-demographic factors among men and women in Myanmar, 2015-16

\begin{tabular}{|c|c|c|c|c|c|c|c|c|c|c|}
\hline & \multicolumn{5}{|l|}{ MEN } & \multicolumn{5}{|l|}{ WOMEN } \\
\hline & $\begin{array}{l}\text { Tobacco } \\
\text { use }\end{array}$ & $\begin{array}{l}\text { Betel } \\
\text { quid }\end{array}$ & $\begin{array}{l}\text { Non- } \\
\text { user }\end{array}$ & $\begin{array}{l}\text { tobacco or betel } \\
\text { quid }\end{array}$ & $\begin{array}{l}\text { Dual } \\
\text { user }\end{array}$ & $\begin{array}{l}\text { Tobacco } \\
\text { use }\end{array}$ & $\begin{array}{l}\text { Betel } \\
\text { quid }\end{array}$ & $\begin{array}{l}\text { Non- } \\
\text { user }\end{array}$ & $\begin{array}{l}\text { tobacco or betel } \\
\text { quid }\end{array}$ & $\begin{array}{l}\text { Dual } \\
\text { user }\end{array}$ \\
\hline Overall & 40.9 & 58.9 & 24.6 & 50.4 & 25.0 & 3.7 & 18.2 & 80.1 & 17.9 & 2.04 \\
\hline \multicolumn{11}{|l|}{ Age groups } \\
\hline $15-20$ & 24.4 & 41.4 & 50.3 & 33.6 & 16.1 & 0.5 & 5.7 & 93.9 & 5.9 & 0.2 \\
\hline $21-30$ & 42.8 & 64.2 & 20.7 & 51.6 & 27.7 & 1.7 & 13.0 & 86.2 & 13.0 & 0.9 \\
\hline $31-40$ & 44.7 & 62.8 & 19.6 & 53.3 & 27.1 & 4.3 & 23.1 & 75.1 & 22.3 & 2.6 \\
\hline $41-49$ & 47.2 & 61.8 & 15.1 & 60.8 & 24.1 & 8.2 & 27.6 & 68.7 & 27.2 & 4.2 \\
\hline \multicolumn{11}{|l|}{ Education $x$} \\
\hline No education & 50.8 & 50.8 & 21.8 & 53.2 & 25.1 & 9.8 & 28.1 & 66.6 & 28.8 & 4.6 \\
\hline Primary & 41.5 & 66.3 & 17.1 & 55.7 & 27.2 & 4.9 & 24.7 & 73.1 & 23.9 & 3.0 \\
\hline Secondary & 37.6 & 56.4 & 29.5 & 46.1 & 24.3 & 1.0 & 11.2 & 88.4 & 10.9 & 0.6 \\
\hline Higher & 30.7 & 51.6 & 38.5 & 45.9 & 18.2 & 0.4 & 4.6 & 95.0 & 5.0 & - \\
\hline \multicolumn{11}{|l|}{ Marital status } \\
\hline Never married & 31.5 & 50.0 & 37.7 & 42.3 & 20.0 & 0.9 & 7.3 & 92.0 & 7.5 & 0.4 \\
\hline Currently married & 43.6 & 64.0 & 17.7 & 55.3 & 27.0 & 4.8 & 22.8 & 74.8 & 22.4 & 2.7 \\
\hline $\begin{array}{l}\text { Divorced/Separated/ } \\
\text { Widowed }\end{array}$ & 69.5 & 55.5 & 16.0 & 40.9 & 43.0 & 6.2 & 30.9 & 67.0 & 28.7 & 4.2 \\
\hline \multicolumn{11}{|l|}{ Wealth Index } \\
\hline Poorest & 45.7 & 64.8 & 16.8 & 54.0 & 29.1 & 10.2 & 35.0 & 60.6 & 33.6 & 5.8 \\
\hline Poorer & 41.0 & 62.1 & 21.3 & 51.7 & 26.9 & 5.0 & 22.8 & 75.0 & 22.3 & 2.7 \\
\hline Middle & 39.3 & 59.5 & 24.4 & 51.3 & 24.2 & 2.9 & 16.2 & 82.3 & 16.3 & 1.4 \\
\hline Richer & 38.0 & 56.1 & 27.9 & 49.1 & 23.0 & 1.5 & 12.4 & 86.7 & 12.6 & 0.7 \\
\hline Richest & 36.9 & 52.9 & 31.7 & 46.1 & 22.0 & 0.6 & 8.2 & 91.5 & 8.2 & 0.3 \\
\hline \multicolumn{11}{|l|}{ Type of residence } \\
\hline Rural & 39.4 & 59.7 & 23.7 & 51.9 & 24.4 & 46.8 & 20.4 & 77.4 & 19.8 & 2.7 \\
\hline Urban & 41.7 & 57.1 & 27.0 & 46.6 & 26.4 & 1.1 & 12.8 & 86.4 & 13.2 & 0.4 \\
\hline \multicolumn{11}{|l|}{ Occupation } \\
\hline $\begin{array}{l}\text { Unemployed/domestic } \\
\text { work }\end{array}$ & 24.4 & 29.8 & 59.8 & 25.6 & 14.5 & 3.5 & 18.9 & 79.7 & 17.8 & 2.3 \\
\hline $\begin{array}{l}\text { Professional/service/ } \\
\text { sales }\end{array}$ & 39.3 & 57.3 & 27.0 & 48.9 & 24.1 & 2.1 & 16.9 & 82.0 & 16.7 & 1.3 \\
\hline Agricultural work & 38.6 & 60.3 & 23.6 & 50.8 & 25.6 & 4.6 & 22.9 & 74.6 & 23.2 & 0.22 \\
\hline Manual worker & 43.0 & 62.3 & 20.0 & 53.9 & 26.1 & 4.5 & 16.6 & 81.1 & 16.5 & 2.4 \\
\hline
\end{tabular}

Chi square test was used to test the statistical significance of differences in tobacco use, betel quid chewing and dual use by sociodemographic factors all factors were statistically significant at $p<0.001$ or $p<0.01$ (betel quid by type of residence) except for tobacco use type of residence $(p>0.05$ )

Men from poor and poorest households had higher odds of being users of one product only as well as 'dual users' (adj. OR 1.4 to 2.2).

Among women being users of one product only and 'dual use' was associated with marital status, wealth index and occupation. Being currently married was protective for being tobacco user and/or betel quid chewer. Women currently married had a lower odds of being betel quid chewer (adj. OR 0.42, 95\% CI 0.3, 0.5) and 'dual users' (adj. OR 0.3, 95\% CI 0.2, 0.7). As compared to women who were manual workers all other occupations had a higher odds of being betel quid chewer (adj. OR 1.5 to 1.7 ) whereas women in the occupational group professional/service/sales had 1.5 times higher odds of being 'dual users.' Wealth index was strongly associated with being tobacco user and/or betel quid chewer with higher effect size than that for men. As compared to women from richest households, women in richer to poorest households had 1.7-7.3 times higher odds of being users of being betel quid chewer; 3.5-32.4 
Table 4 Factors associated with tobacco use and betel quid chewing by multinomial regression analyses

\begin{tabular}{|c|c|c|c|c|c|c|c|c|c|c|c|c|}
\hline & \multicolumn{6}{|l|}{ MEN } & \multicolumn{6}{|c|}{ WOMEN } \\
\hline & \multicolumn{2}{|c|}{$\begin{array}{l}\text { Betel quid chewer } \\
\text { versus non-user }\end{array}$} & \multicolumn{2}{|c|}{$\begin{array}{l}\text { Tobacco user' } \\
\text { versus non-user }\end{array}$} & \multicolumn{2}{|c|}{$\begin{array}{l}\text { dual user' versus } \\
\text { non-user }\end{array}$} & \multicolumn{2}{|c|}{$\begin{array}{l}\text { Betel quid } \\
\text { chewer versus } \\
\text { non-user }\end{array}$} & \multicolumn{2}{|c|}{$\begin{array}{l}\text { Tobacco user' } \\
\text { versus non-user }\end{array}$} & \multicolumn{2}{|c|}{$\begin{array}{l}\text { dual user' versus } \\
\text { non-user }\end{array}$} \\
\hline & $\begin{array}{l}\text { Adj. Odds } \\
\text { Ratio } \\
(95 \% \mathrm{Cl})\end{array}$ & $\overline{p \text {-value }}$ & $\begin{array}{l}\text { Adj. Odds } \\
\text { Ratio } \\
(95 \% \mathrm{Cl})\end{array}$ & $\overline{p \text {-value }}$ & $\begin{array}{l}\text { Adj. Odds } \\
\text { Ratio } \\
(95 \% \mathrm{Cl})\end{array}$ & $\overline{p \text {-value }}$ & $\begin{array}{l}\text { Adj. } \\
\text { Odds } \\
\text { Ratio } \\
(95 \% \\
\text { Cl) }\end{array}$ & $\overline{p \text {-value }}$ & $\begin{array}{l}\text { Adj. Odds } \\
\text { Ratio } \\
(95 \% \mathrm{Cl})\end{array}$ & $\overline{p \text {-value }}$ & $\begin{array}{l}\text { Adj. Odds } \\
\text { Ratio } \\
(95 \% \mathrm{Cl})\end{array}$ & p-value \\
\hline Age & $\begin{array}{l}1.0(1.0, \\
1.1)\end{array}$ & $<0.001$ & $\begin{array}{l}1.1(1.0 \\
1.1)\end{array}$ & $<0.001$ & $\begin{array}{l}1.0(1.0 \\
1.1)\end{array}$ & $<0.001$ & $\begin{array}{l}1.1(1.0 \\
1.1)\end{array}$ & $<0.001$ & $\begin{array}{l}1.1(1.1 \\
1.2)\end{array}$ & $<0.001$ & $\begin{array}{l}1.1(1.1 \\
1.2)\end{array}$ & $<0.001$ \\
\hline \multicolumn{13}{|l|}{ Marital status } \\
\hline $\begin{array}{l}\text { Divorced/ } \\
\text { separated/ } \\
\text { widowed }\end{array}$ & 1 & & 1 & & 1 & & 1 & & 1 & & 1 & \\
\hline $\begin{array}{l}\text { Currently } \\
\text { married }\end{array}$ & $\begin{array}{l}1.5(0.7 \\
3.4)\end{array}$ & 0.271 & $\begin{array}{l}0.4(0.2, \\
0.9)\end{array}$ & 0.028 & $\begin{array}{l}0.3(0.2, \\
0.7)\end{array}$ & 0.002 & $\begin{array}{l}0.4(0.3, \\
0.5)\end{array}$ & $<0.001$ & $\begin{array}{l}1.0(0.5 \\
2.2)\end{array}$ & 0.986 & $\begin{array}{l}0.3(0.2, \\
0.7)\end{array}$ & 0.001 \\
\hline Never married & $\begin{array}{l}2.1(1.0, \\
4.5)\end{array}$ & 0.049 & $\begin{array}{l}0.5(0.3 \\
1.2)\end{array}$ & 0.111 & $\begin{array}{l}0.5(0.3 \\
1.1)\end{array}$ & 0.089 & $\begin{array}{l}0.8(0.6 \\
0.9)\end{array}$ & 0.016 & $\begin{array}{l}1.4(0.8, \\
2.6)\end{array}$ & 0.272 & $\begin{array}{l}0.8(0.5 \\
1.2)\end{array}$ & 0.227 \\
\hline \multicolumn{13}{|l|}{ Wealth Index } \\
\hline Richest & 1 & & 1 & & 1 & & 1 & & 1 & & 1 & \\
\hline Richer & $\begin{array}{l}1.2(0.9 \\
1.7)\end{array}$ & 0.291 & $\begin{array}{l}1.2(0.8 \\
1.7)\end{array}$ & 0.448 & $\begin{array}{l}1.2(0.8 \\
1.72)\end{array}$ & 0.403 & $\begin{array}{l}1.7(1.3 \\
2.2)\end{array}$ & $<0.001$ & $\begin{array}{l}3.5(1.7 \\
7.3)\end{array}$ & 0.001 & $\begin{array}{l}2.4(1.1 \\
5.4)\end{array}$ & 0.036 \\
\hline Middle & $\begin{array}{l}1.4(0.9 \\
2.0)\end{array}$ & 0.081 & $\begin{array}{l}1.3(0.8 \\
1.9)\end{array}$ & 0.259 & $\begin{array}{l}1.4(0.9 \\
2.1)\end{array}$ & 0.124 & $\begin{array}{l}2.4(1.8 \\
3.0)\end{array}$ & $<0.001$ & $\begin{array}{l}6.2(2.9 \\
13.4)\end{array}$ & $<0.001$ & $\begin{array}{l}5.0(2.3 \\
10.9)\end{array}$ & $<0.001$ \\
\hline Poorer & $\begin{array}{l}1.6(1.1 \\
2.3)\end{array}$ & 0.025 & $\begin{array}{l}1.4(0.9 \\
2.1)\end{array}$ & 0.141 & $\begin{array}{l}1.6(1.1 \\
2.5)\end{array}$ & 0.020 & $\begin{array}{l}3.6(2.8, \\
4.6)\end{array}$ & $<0.001$ & $\begin{array}{l}11.3(5.4 \\
23.8)\end{array}$ & $<0.001$ & $\begin{array}{l}11.6(5.3 \\
25.3)\end{array}$ & $<0.001$ \\
\hline Poorest & $\begin{array}{l}1.9(1.2, \\
3.0)\end{array}$ & 0.005 & $\begin{array}{l}1.8(1.2, \\
2.9)\end{array}$ & 0.007 & $\begin{array}{l}2.2(1.4 \\
3.5)\end{array}$ & 0.001 & $\begin{array}{l}7.3(5.5 \\
9.6)\end{array}$ & $<0.001$ & $\begin{array}{l}32.4(15.3, \\
68.6)\end{array}$ & $<0.001$ & $\begin{array}{l}34.4(15.9, \\
74.6)\end{array}$ & $<0.001$ \\
\hline \multicolumn{13}{|l|}{ Occupation } \\
\hline $\begin{array}{l}\text { Manual } \\
\text { worker }\end{array}$ & 1 & & 1 & & 1 & & 1 & & 1 & & 1 & \\
\hline $\begin{array}{l}\text { Professional/ } \\
\text { service/ sales }\end{array}$ & $0.3(0.2,0.4)$ & $<0.001$ & $\begin{array}{l}0.4(0.3, \\
0.7)\end{array}$ & 0.001 & $0.3(0.2,0.6)$ & 0.000 & $\begin{array}{l}1.5(1.2 \\
1.9)\end{array}$ & $<0.001$ & $\begin{array}{l}0.9(0.6 \\
1.3)\end{array}$ & 0.587 & $\begin{array}{l}1.5(1.0, \\
2.1)\end{array}$ & 0.037 \\
\hline $\begin{array}{l}\text { Agricultural } \\
\text { work }\end{array}$ & $\begin{array}{l}0.8(0.6 \\
1.1)\end{array}$ & 0.108 & $\begin{array}{l}0.7(0.5 \\
1.1)\end{array}$ & 0.123 & $\begin{array}{l}0.8(0.6 \\
1.1)\end{array}$ & 0.210 & $\begin{array}{l}1.7(1.3 \\
2.1)\end{array}$ & $<0.001$ & $\begin{array}{l}0.9(0.6, \\
1.6)\end{array}$ & 0.828 & $\begin{array}{l}1.1(0.7, \\
1.8)\end{array}$ & 0.662 \\
\hline $\begin{array}{l}\text { Unemployed/ } \\
\text { domestic } \\
\text { work }\end{array}$ & $\begin{array}{l}0.8(0.6 \\
1.0)\end{array}$ & 0.065 & $\begin{array}{l}0.7(0.5 \\
0.9)\end{array}$ & 0.019 & $\begin{array}{l}0.7(0.5 \\
1.0)\end{array}$ & 0.045 & $\begin{array}{l}1.5(1.1 \\
1.92)\end{array}$ & 0.008 & $\begin{array}{l}1.1(0.7, \\
1.7)\end{array}$ & 0.794 & $\begin{array}{l}0.9(0.6, \\
1.5)\end{array}$ & 0.782 \\
\hline $\begin{array}{l}\text { Exposure to } \\
\text { media }\end{array}$ & $\begin{array}{l}1.0(0.9 \\
1.2)\end{array}$ & 0.960 & $\begin{array}{l}0.8(0.7 \\
0.9)\end{array}$ & 0.017 & $\begin{array}{l}1.0(0.9 \\
1.2)\end{array}$ & 0.858 & $\begin{array}{l}1.0(0.9 \\
1.1)\end{array}$ & 0.450 & $\begin{array}{l}1.0(0.8, \\
1.1)\end{array}$ & 0.642 & $\begin{array}{l}0.9(0.8, \\
1.1)\end{array}$ & 0.274 \\
\hline
\end{tabular}

'Dual user'- tobacco user as well as betel quid chewer

times higher odds of being tobacco user. Likewise compared to women from richest households, women in richer to poorest households had 2.4-34.4 times higher odds of being 'dual users.' There was a clear gradient across the wealth groups the odds of being tobacco user and/or betel quid chewer increased from the richest to the poorest.

\section{Discussion}

Overall national-level estimates of tobacco use, betel quid chewing, and dual usage is a first comprehensive report for Myanmar. We found that prevalence of betel quid chewing was higher than tobacco use in both sexes. Tobacco use $(41.7 \%$ vs. $5.1 \%)$ and betel quid chewing (58.7\% vs. $20.7 \%)$ was higher among men than women. SLT use was very low; hence, most tobacco users smoked cigarettes and/or cheroots. Nearly half the men and fifth of women either used tobacco products or chewed betel quid. Notably, a quarter of the men were 'dual users.' 'Dual use' was associated with increasing age, lower wealth and lower education in both sexes; the effect sizes of these associations were higher among 
women than men indicating that wider socio-economic differentials in tobacco use and betel quid chewing exist among women.

This national report is helpful for monitoring of the tobacco use pattern over the coming years and for comparisons with previous sub national studies in Myanmar and those with neighbouring countries. Prevalence of tobacco smoking and betel quid chewing among men and women in Myanmar are reported in WHO-STEPS survey (2009) [28] and National Survey on Diabetes Mellitus and Risk Factors for Non communicable Disease (2014) [29]. The reported prevalence rates in these surveys are comparable to Myanmar DHS estimates. Other survey reports available for Myanmar are Sentinel Prevalence Studies of Tobacco in years 2001, 2004 \& 2007 which showed that prevalence of tobacco smoking was decreasing from years 2001 through 2007 but smokeless tobacco use (inclusive of betel quid) increased during the same time period [30]. Betel quid use rates among men and women in Myanmar DHS was higher than Nepalese men $(58.7 \%$ vs. $43.6 \%)$ and Indonesian women (20.7\% vs. $46.8 \%$ ) reported in Asian Betel-Quid Consortium Study 2009-10 [8]. Previous reports from SA and SEA $[6,16]$ have shown that smoking, tobacco use and dual tobacco use varied among the countries; smoking and smokeless tobacco use rates in Myanmar are comparable to India, Pakistan and Nepal $[6,16]$. Overall, tobacco smoking rates among men are comparable to neighbouring countries [31] but rates among women are increasing; yet the disparity between men and women was as high as in other SA countries [32]. Prevalence of 'dual use' among Myanmar men is much higher than men from other Asian countries [16]. Reports from other Asian countries, do not include betel quid to define 'dual use'. In Myanmar, being an older adult, poor, less educated, currently married and manual workers had higher odds of 'dual use' as in a previous studies $[6,16]$.

Myanmar tobacco control program framed in 2000 focussed on smoking tobacco products only. Tobacco control was initiated in 2004 when Myanmar ratified the WHO-FCTC [33] and Myanmar Tobacco Control Law of 2006 covers all forms of tobacco consumption [34]; yet tobacco control in Myanmar is still in very early stages [35]. Considering an overall prevalence which has even shown an increase [31] and widespread prevalence of betel quid chewing [13], Myanmar is unlikely to achieve its Global Action Plan' target 5 of 30\% relative reduction of current tobacco use among adults aged 15 years and above by 2025 [36]. Myanmar faces an unprecedented challenge of 'dual use' of tobacco use (smoking and smokeless tobacco) and betel quid chewing. Antismoking measures such as smoking ban in public places and prohibition of tobacco product advertising and promotion, health warnings on tobacco packages were implemented after a long delay of WHO-FCTC ratification and passing of tobacco control law [37, 38]. In our analyses mass media exposure was not associated with tobacco use or betel quid chewing possibly the associated was masked by wealth and education strongly associated with tobacco use. Betel quid (areca nut, catechu, slaked lime often mixed with tobacco leaves) is a deeply rooted in the culture and tradition of Myanmar is often consumed during ceremonial occasions such as weddings and offered to guests visiting homes. Myanmar people believe that betel quid is not as harmful as smoking and chewing raw tobacco among rural folks is common who believe that tobacco leaves are breathfreshening and mouth-cleansing [14]. Low cost, manufactured, sales, and marketing mainly by the unorganised sector makes smokeless tobacco more challenging to regulate [13].

This is a first comprehensive report on a nationally representative sample providing the most current estimates of tobacco use and betel quid chewing in Myanmar. National surveys done in Myanmar [29, 31, 35], did not provide detailed analyses of distribution and social determinants of tobacco smoking, SLT use and betel quid chewing. Tobacco use prevalence estimates by sex are comparable with other DHS-based estimates from the region $[6,16]$ and our estimates will serve as a bench mark for monitoring the progress towards Global non-communicable disease prevention target achievement [36] and for the global tobacco surveillance system [39].

Our results should be interpreted in the light of some limitations of DHS data arising from the survey design and questionnaire contents. Firstly, our prevalence among adults aged 15-49 would be an underestimate, if those aged $>49$ years had higher rates of tobacco use. Secondly, the DHS questionnaire had limited questions covering current use only; this did not allow us to estimate the detailed indicators such as former use, age at initiation, and quit attempts. Thirdly, studies about SLT use in Myanmar have reported about several chewable products which may or may not have been included betel quid chewing $[5,14]$. However, Myanmar DHS asked a separate question about betel quid chewing without details about mixing betel quid with tobacco; hence, we could not report betel quid chewing as SLT, instead we reported it as a separate behaviour. A recent study has reported that about $84 \%$ of the betel quid chewers used tobacco along with it [40]. As a result, our estimates on SLT were likely underestimated because DHS survey questions did not ask about the tobacco leaves consumed along with betel quid. Fourthly, DHS being a cross-sectional in design, temporality of reported associations cannot be inferred. Fifthly, association of 
'dual use' versus either use and non-use with occupation was in opposite directions between men and women. Possible reasons for this could be misclassification of occupational groups and/or a residual confounding. Lastly, tobacco use being a self-report, we cannot rule out its under-reporting in conservative societies of Asia. DHS did not verify self-reported tobacco use by measuring urinary cotinine levels.

Sequential national-level surveys are needed to monitor socio-economic equalities in tobacco use and betel quid chewing and assist policies and control programs targeting vulnerable groups. Survey questionnaires should include items to identify both current and former users, cessation behaviors and inquire about mixing tobacco leaves into the betel quid which is a common practice in Myanmar. A high proportion of 'dual users' of tobacco and betel quid warrants an urgent need for health screening and provide cessation counselling especially for 'dual users.'

\section{Conclusions}

Tobacco use prevalence rate is alarmingly high among Myanmar men. Betel quid chewing rate is also very high among men and to a lesser extent among women. Stricter implementation and effective enforcement of existing tobacco control measures is necessary particularly among the least educated, and poorer households. Regular Surveys covering tobacco use and MPOWER indicators are needed to monitor tobacco use and betel quid chewing and the implementation of tobacco control strategies.

\section{Abbreviations}

DHS: Demographic and Health Survey; SA: South Asia; SEA: South-east Asia; SLT: Smokeless tobacco; FCTC: Framework Convention on Tobacco Control; MoHS: Ministry of Health and Sports; 3MDG: Three Millennium Development Goals; ICF: Inner City Fund; PSU: Primary Sampling Unit; EA: Enumeration Areas; ORC: Opinion Research Corporation; OR: Odds Ratio; MPOWER: Monitoring, Protection, Offering, Warning, Enforcing, Raising; WHO: World Health Organisation; STEPS: STEP-wise approach to surveillance

\section{Acknowledgments}

We thank the measuredhs for providing data access to Myanmar

Demographic and health survey 2015-16.

\section{Authors' contributions}

CTS conceptualised the paper, performed the data analysis, made substantial revisions of entire manuscript with inputs from SNA and SPV. SNA prepared the draft results section, and tables. SPV, wrote first draft of background and methods. All authors read and approved the final manuscript.

\section{Funding}

No funding was received to write this report.

\section{Availability of data and materials}

The data of Myanmar Demographic and Health Survey are available from measuredhs website and were provided to the authors up on a written request. The files used for analyses and writing this manuscript are available from the authors up on request.

\section{Ethics approval and consent to participate}

The institutional review boards of ORC Macro International Inc. and participating institutions in Myanmar provided the ethical clearance. Before each interview, details of the survey were explained, and informed consent was obtained. Secondary data analyses ethical review was not required since the data used for analyses was de-identified.

\section{Consent for publication}

Not applicable.

\section{Competing interests}

The authors declare that they have no competing interests.

\section{Author details}

'Department of Community Medicine, International Medical University, Bukit Jalil, 57000 Kuala Lumpur, Malaysia. ²Department of Pathology, International Medical University, Bukit Jalil, 57000 Kuala Lumpur, Malaysia.

Received: 2 July 2020 Accepted: 27 January 2021

Published online: 03 February 2021

\section{References}

1. Kyu HH, Abate D, Abate KH, Abay SM, Abbafati C, Abbasi N, et al. Global, regional, and national disability-adjusted life-years (DALYs) for 359 diseases and injuries and healthy life expectancy (HALE) for 195 countries and territories, 1990-2017: a systematic analysis for the global burden of disease study 2017. Lancet. 2018;392:1859-922.

2. Reitsma MB, Fullman N, Ng M, Salama JS, Abajobir A, Abate KH, et al. Smoking prevalence and attributable disease burden in 195 countries and territories, 1990-2015: a systematic analysis from the global burden of disease study 2015. Lancet. 2017;389:1885-906.

3. Stanaway JD, Afshin A, Gakidou E, Lim SS, Abate D, Abate KH, et al. Global, regional, and national comparative risk assessment of 84 behavioural, environmental and occupational, and metabolic risks or clusters of risks for 195 countries and territories, 1990-2017: a systematic analysis for the global burden of disease study 2017. Lancet. 2018;392:1923-94.

4. World Health Organization: WHO global report on trends in prevalence of tobacco smoking 2000-2025. 2018.

5. Sinha DN, Gupta PC, Ray CS, Singh PK. Prevalence of smokeless tobacco use among adults in WHO South-East Asia. Indian J Cancer. 2012;49:342.

6. Sreeramareddy CT, Pradhan PMS, Mir IA, Sin S. Smoking and smokeless tobacco use in nine south and southeast Asian countries: prevalence estimates and social determinants from demographic and health surveys. Popul Health Metrics. 2014;12:22

7. Sinha DN, Abdulkader RS, Gupta PC. Smokeless tobacco-associated cancers: a systematic review and meta-analysis of Indian studies. Int J Cancer. 2016; 138:1368-79.

8. Lee CH, Min-Shan Ko A, Warnakulasuriya S, Ling TY, Rajapakse PS, Zain RB, et al. Population burden of betel quid abuse and its relation to oral premalignant disorders in south, southeast, and East Asia: an Asian betelquid consortium study. Am J Public Health. 2012;102:e17-24.

9. Anand R, Dhingra C, Prasad S, Menon I. Betel nut chewing and its deleterious effects on oral cavity. J Cancer Res Ther. 2014;10:499.

10. Lee YA, Li S, Chen Y, Li Q, Chen C, Hsu W, et al. Tobacco smoking, alcohol drinking, betel quid chewing, and the risk of head and neck cancer in an east Asian population. Head Neck. 2019;41:92-102.

11. Mizukawa N, Win SS, Thein ZM, Htwe MT, Yoshioka Y, Kimata Y, et al. The incidence of Oral and Oropharyngeal cancers in betel quid-chewing populations in South Myanmar rural areas. Acta Med Okayama. 2017;71: 519-24

12. Zaw KK, Ohnmar M, Hlaing MM, Win SS, Aye PP, Shwe S, et al. Betel quid and oral potentially malignant disorders in a periurban township in Myanmar. PLoS One. 2016;11:e0162081.

13. Sein T, Swe T, Toe MM, Zaw KK, Sein TO. Challenges of smokeless tobacco use in Myanmar. Indian J Cancer. 2014;51:3.

14. Kyaing NN, Sein T, Sein AA, Htike MT, Tun A, Shein NNN. Smokeless tobacco use in Myanmar. Indian J Cancer. 2012;49:347.

15. Framework Convention on Tobacco Control. https://www.who.int/fctc/en/. Accessed September 5th, 2020.

16. Sinha DN, Suliankatchi RA, Amarchand R, Krishnan A. Prevalence and sociodemographic determinants of any tobacco use and dual use in six 
countries of the WHO South-East Asia region: findings from the demographic and health surveys. Nicotine Tob Res. 2016;18:750-6.

17. Song $H$, Wan $Y, X u$ YY. Betel quid chewing without tobacco: a meta-analysis of carcinogenic and precarcinogenic effects. Asia Pac J Public Health. 2015; 27:NP47-57.

18. World Health Organization. WHO report on the global tobacco epidemic, 2017: monitoring tobacco use and prevention policies. Geneva: World Health Organization; 2017.

19. Mini GK, Thankappan KR. Switching to smokeless tobacco, the most common smoking cessation method: results from the global adult tobacco survey, India. Public health. 2016;136:172.

20. Suliankatchi RA, Sinha DN, Rath R, Aryal KK, Zaman MM, Gupta PC, et al. Smokeless tobacco use is "replacing" the smoking epidemic in the SouthEast Asia region. Nicotine Tob Res. 2017;21:95-100.

21. Myint SK, Narksawat K, Sillabutra J. Prevalence and factors influencing betel nut chewing among adults in west insein township, Yangon, Myanmar. Southeast Asian J Trop Med Public Health. 2016:47:1089-97.

22. Zaw KK, Ohnmar M, Hlaing MM, Win SS, Than Htike MM, Aye PP. Betel quid chewing in Dagon (east) township in 2013. In: In a paper presented at the Myanmar Medical Research Congress; 2014.

23. Ministry of Health and Sports (MoHS): Myanmar Demographic and Health Survey 2015-2016. 2017.

24. Corsi DJ, Neuman M, Finlay JE, Subramanian SV. Demographic and health surveys: a profile. Int J Epidemiol. 2012:41:1602-13.

25. Measure DHS. Demographic and health surveys. Calverton: Measure DHS; 2013.

26. Rutstein SO, Staveteig S. Making the demographic and health surveys wealth index comparable; 2014

27. Vyas S, Kumaranayake L. Constructing socio-economic status indices: how to use principal components analysis. Health Policy Plan. 2006;21:459-68.

28. World Health Organization. Noncommunicable disease risk factory survey, Myanmar 2009. New Delhi: WHO regional office for south-East Asia; 2012.

29. Department of Public Health and Department of Medical Research RGoM Report on National Survey on Diabetes Mellitus and Risk Factors for Non communicable Disease in Mynamar. 2019.

30. Ministry of Health, Government of Myanmar. Brief Profile on Tobacco Control in Myanmar. 2019.

31. Sinha DN, Bhartiya D, Kumar A, Singh H, Mehrotra R. Men in Myanmar submerged in tobacco: women following. Nicotine Tob Res. 2016;19:13978.

32. Pampel FC. Global patterns and determinants of sex differences in smoking. Int J Comp Sociol. 2006;47:466-87.

33. World Health Organisation. WHO FCTC Implementation Database, Myanmar factsheet. 2019.

34. The New Light of Myanmar. The Control of Smoking and Consumption of Tobacco Product Law (State Peace and Development Council Law No. 5/ 2006); Friday, 5 May 2006. 5-5-2006.

35. Grover S, Sinha DN, Gupta S, Gupta PC, Mehrotra R. The changing face of risk factors for non-communicable disease in Myanmar: findings from the 2009 and 2014 WHO STEP surveys. J Public Health (Oxf). 2018.

36. World Health Organization. WHO. Global Action Plan for the Prevention and Control of NCDs 2013-2020 [Internet]. WHA66. Geneva: World Health Organization; 2013. p. 55. [cited 2014 Jan 27].

37. The Government of the Republic of the Union of Myanmar, TheMinistry of Health. Notification No. 6/2014. The 3rd Waxing of Tabaung, 1375 M.E. 3-42014.

38. The Government of the Republic of the Union of Myanmar. Ministry of Health. Proclamation No. 11/2016. Myanmar Year 1377. 6th of Tabodwe Month (29th February 2016). 29-2-2016.

39. Warren CW, Lee J, Lea V, Goding A, O'hara B, Carlberg M, et al. Evolution of the global tobacco surveillance system (GTSS) 1998-2008. Glob Health Promot. 2009;16:4-37.

40. Papke RL, Bhattacharyya I, Hatsukami DK, Moe I, Glatman S. Betel nut (areca) and smokeless tobacco use in Myanmar. Substance Use Misuse. 2020;55(9): 1385-94.

\section{Publisher's Note}

Springer Nature remains neutral with regard to jurisdictional claims in published maps and institutional affiliations.

Ready to submit your research? Choose BMC and benefit from:

- fast, convenient online submission

- thorough peer review by experienced researchers in your field

- rapid publication on acceptance

- support for research data, including large and complex data types

- gold Open Access which fosters wider collaboration and increased citations

- maximum visibility for your research: over $100 \mathrm{M}$ website views per year

At BMC, research is always in progress.

Learn more biomedcentral.com/submissions 